\title{
Spherical Aberration Correction Using Refractive-Diffractive Lenses with an Analytic-Numerical Method
}

\author{
Sergio Vázquez-Montiel, Omar García-Liévanos, and Juan Alberto Hernández-Cruz
}

Marginal Meridional Rays, Instituto Nacional de Astrofísica Óptica y Electrónica, Apdo. Postal 51 y 216, Puebla-72000, Mexico

Correspondence should be addressed to Omar García-Liévanos, ogarcial@ipn.mx

Received 19 February 2010; Accepted 14 July 2010

Academic Editor: Michael Fiddy

Copyright (C) 2010 Sergio Vázquez-Montiel et al. This is an open access article distributed under the Creative Commons Attribution License, which permits unrestricted use, distribution, and reproduction in any medium, provided the original work is properly cited.

\begin{abstract}
We propose an alternative method to design diffractive lenses free of spherical aberration for monochromatic light. Our method allows us to design diffractive lenses with the diffraction structure recorded on the last surface; this surface can be flat or curved with rotation symmetry. The equations that we propose calculate the diffraction profiles for any substratum, for any f-number, and for any position of the object. We use the lens phase coefficients to compensate the spherical aberration. To calculate these coefficients, we use an analytic-numerical method. The calculations are exact, and the optimization process is not required.
\end{abstract}

\section{Introduction}

Spherical aberration is, in many cases, the most important of all primary aberrations, because it affects the whole field of the lens, including the vicinity of the optical axis. It is due to different focus positions for a marginal ray, meridional ray, and paraxial rays. An alternative to minimize the spherical aberration is to use diffractive optical elements (DOE). Diffractive lenses are essentially gratings with a variable spacing groove which introduces a chromatic aberration that is worse than conventional refractive/reflective optical elements. In some applications, an optical component may require a diffractive surface combined with a classic lens element. By using the diffractive properties, it is possible to design hybrid elements to obtain an achromatically corrected element [1]. In other cases, the requirements can be satisfied by just using a diffractive element. In general, iterative methods are used to design these lenses [2]. Also, some people have used analytical third-order and numerical integrator methods to design diffractive lenses $[1,3]$. The diffractive lenses we describe in this paper are limited to monochromatic applications; however, our proposed method is valid for a wide range of wavelengths.

We use lens phase coefficients to compensate spherical aberration. To calculate these coefficients, we use an analytic- numerical method. The calculations are exact, simple and quick. A process of optimization is not required.

The manufacturing problem of diffractive lenses is not considered here; to solve this problem you can read CastroRamos et al. [4].

First, we describe the diffractive lenses theory. Also, we give a brief derivation of the general grating equation to trace a couple of light rays through a rotationally symmetrical surface. Then, we establish the analytic-numerical method to minimize spherical aberration. We propose some heights to correct the spherical aberration. Finally, we conclude by providing a design example.

\section{Theory of the Diffractive Lenses}

Diffractive lenses can be described by a polynomial phase function [5]

$$
\phi(x, y)=\frac{2 \pi}{\lambda} \sum_{m} \sum_{n} a_{m n} x^{m} y^{n},
$$

where $\lambda$ is the design wavelength; $a_{m n}$ are the lens phase coefficients; $x, y$ are the coordinates in the diffractive lens. 
We will consider that the diffractive lens is rotationally symmetrical, so (1) is rerewritten as

$$
\phi(y)=\frac{2 \pi}{\lambda}\left(a_{0}+a_{2} y^{2}+a_{4} y^{4}+a_{6} y^{6}+a_{8} y^{8}+\cdots\right) .
$$

Here, the longitudinal displacement of the reference sphere is $a_{0}=0$ because we have assumed it is in the ideal focus. The coefficient $a_{2}$ are implicit lens paraxial properties; it is equal to $-1 / 2 f$, where $f$ is the focal length. The remainder coefficients in (2) give the amount of spherical aberrations of the first- second- and higher-order $[6,7]$.

Designers usually use some commercial optical design programs to obtain the lens phase coefficients by using an optimization process. We will describe an analytical method to obtain these coefficients.

To trace a pencil of rays through the diffractive optical surface, we use the grating equation. For a flat surface, the grating equation is given by

$$
n^{\prime} \sin I^{\prime}-n \sin I=m \lambda f
$$

where $n^{\prime}$ and $n$ are refractive indexes for two different mediums, $I^{\prime}$ and $I$ are the diffractive and incident angles; $f$ is the grating frequency; $m$ is the diffracted order.

To analyze the light propagation through a diffractive curved surface, we have to change the form of the last equation. After some algebra, we obtain the general grating equation

$$
\left(n^{\prime} M_{2}-n M_{1}\right) \cos \theta_{N}+\left(n N_{1}-n^{\prime} N_{2}\right) \sin \theta_{N}=m \lambda \cos \theta_{N} f,
$$

where the direction of refracted and diffracted rays is given by the direction cosines $M_{1}, M_{2}, N_{1}$, and $N_{2}$ as are shown in the Figure 1. This analysis considers that the diffractive lens is rotationally symmetrical, and then the direction cosines $L_{0}=$ $0, L_{1}=0, L_{2}=0 . \theta_{N}$ is the angle between the normal at surface and optical axis, it is given by

$$
\sin \theta_{N}, \cos \theta_{N}=\frac{(\partial F / \partial y, \partial F / \partial z)}{\left\{(\partial F / \partial x)^{2}+(\partial F / \partial y)^{2}+(\partial F / \partial z)^{2}\right\}^{1 / 2}},
$$

here, $F$ is the surface function in which the diffractive lens will be recorded, and $x$ and $y$ are the surface coordinates.

The grating frequency of (3) and (4) can be calculated in one dimension by

$$
\begin{gathered}
f_{y}=\frac{1}{2 \pi} \frac{\partial \phi}{\partial y}, \\
f_{y}=\frac{1}{\lambda}\left[\sum_{k=1}^{\infty} 2 k a_{2 k} y^{2 k-1}\right],
\end{gathered}
$$

where $\phi$ is the phase function given by (2) $k$ is an integer equal to $1,2,3,4, \ldots$, and the diffracted order $m=1$. With this, (4) can be rewritten as

$$
\begin{gathered}
{\left[\left(n^{\prime} M_{2}-n M_{1}\right) \cos \theta_{N}+\left(n N_{1}-n^{\prime} N_{2}\right) \sin \theta_{N}\right]} \\
\quad=\cos \theta_{N}\left[\sum_{k=1}^{\infty} 2 k y^{2 k-1} a_{2 k}\right] .
\end{gathered}
$$

\begin{tabular}{|c|c|c|c|c|}
\hline Surfaces & $\begin{array}{c}\text { Radius } \\
(\mathrm{mm})\end{array}$ & $\begin{array}{c}\text { Thickness } \\
(\mathrm{mm})\end{array}$ & $\begin{array}{l}\text { Radius aperture } \\
(\mathrm{mm})\end{array}$ & Glass \\
\hline 1 & $\infty$ & 200 & & Air \\
\hline 2 & 101.954 & 8.138 & 25 & BK7 \\
\hline 3 DOE & -101.954 & 66.059 & 25 & Air \\
\hline 4 & $\infty$ & 0 & & Air \\
\hline
\end{tabular}

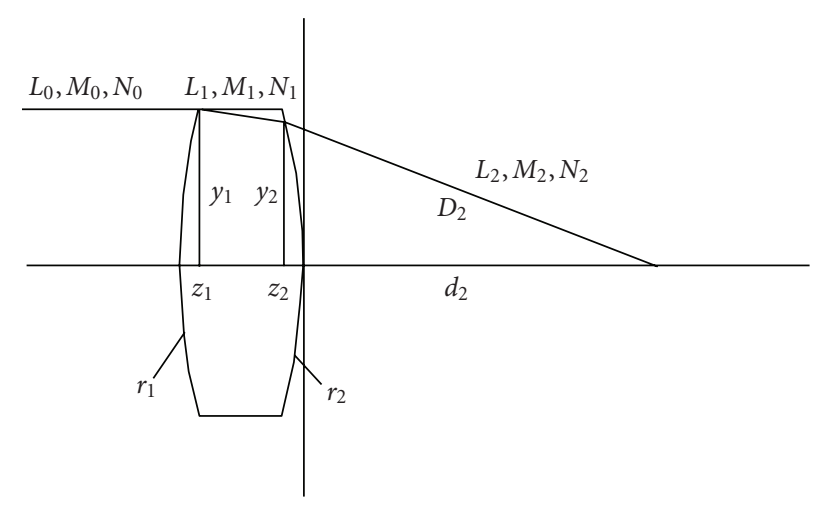

FIGURE 1: Lens parameter. The diffractive surface is on the second surface.

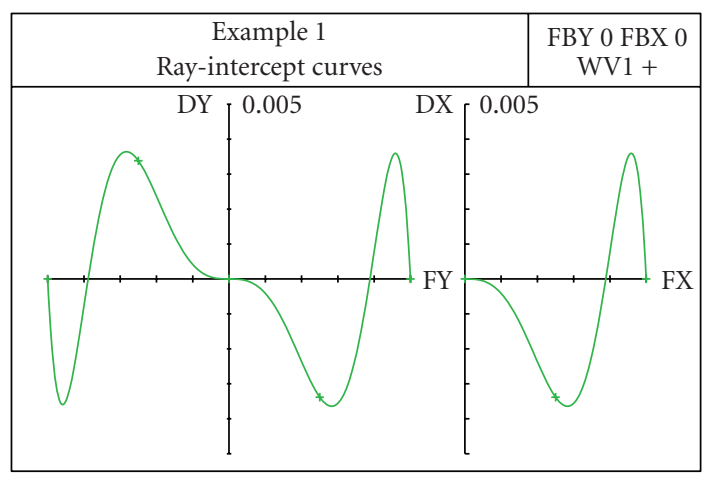

FIgURE 2: Transversal spherical aberration of the example one.

TABLE 1: Example 1 data.

Using (5) and (7), we can trace $n$ rays through the surface at different heights on the pupil. Then they can arrange a $k \times k$ equations system. The number of equations depends of the number of coefficients that we want to find.

Then, if we want to find $k$ coefficients, we need to solve an equation system similar to(8)

$$
\left[\begin{array}{ccccc}
w_{11} & w_{12} & w_{13} & \cdots & w_{1 k} \\
w_{21} & w_{22} & w_{23} & \cdots & w_{2 k} \\
w_{31} & w_{32} & w_{33} & \cdots & w_{3 k} \\
\vdots & \vdots & \vdots & \cdots & \vdots \\
w_{k 1} & w_{k 2} & w_{k 3} & \cdots & w_{k k}
\end{array}\right]\left[\begin{array}{c}
-\frac{1}{2 f} \\
a_{4} \\
a_{6} \\
\vdots \\
a_{2 k}
\end{array}\right]=\left[\begin{array}{c}
A_{0} \\
A_{1} \\
A_{2} \\
\vdots \\
A_{k}
\end{array}\right]
$$

where $w$ represents the different constants of the right side of (7), and $A$ are the constants of the left side of the same equation, all for different height rays on the pupil. 


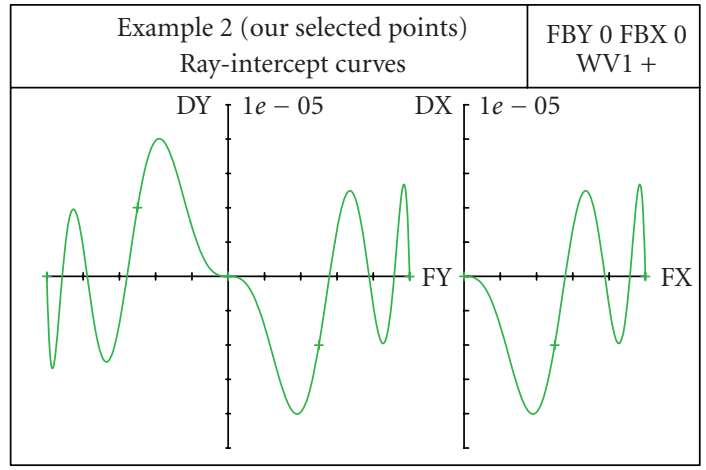

(a)

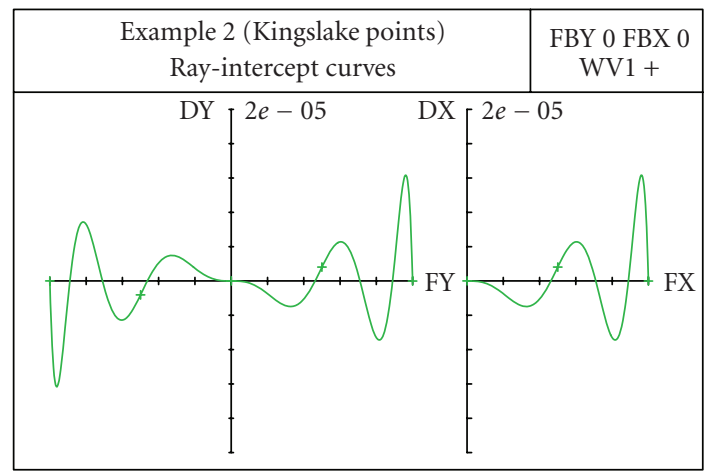

(b)

FIgURE 3: Transversal spherical aberration of the example 2.

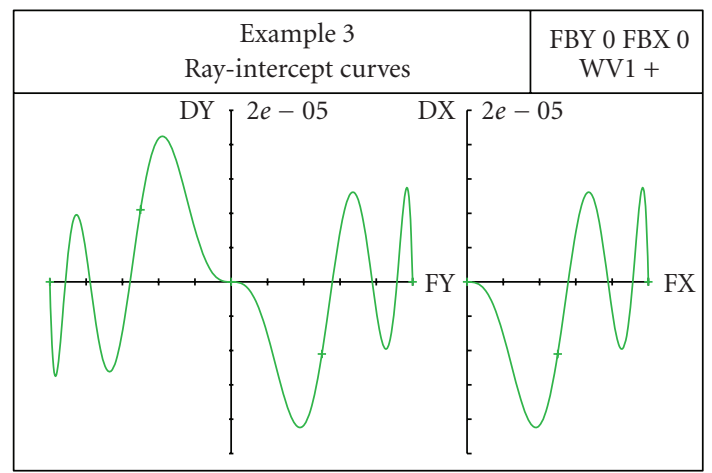

Figure 4: Transversal spherical aberration of the example 3.

\section{How Many and What Heights Should Be Corrected}

The spherical aberration of the ray in any optical system can be expressed as

$$
\frac{\partial W(0, y)}{\partial y}=4 b_{1}\left(y^{3}\right)+6 b_{2}\left(y^{5}\right)+8 b_{3}\left(y^{7}\right)+\cdots
$$

Considering only big f/numbers, the spherical aberration

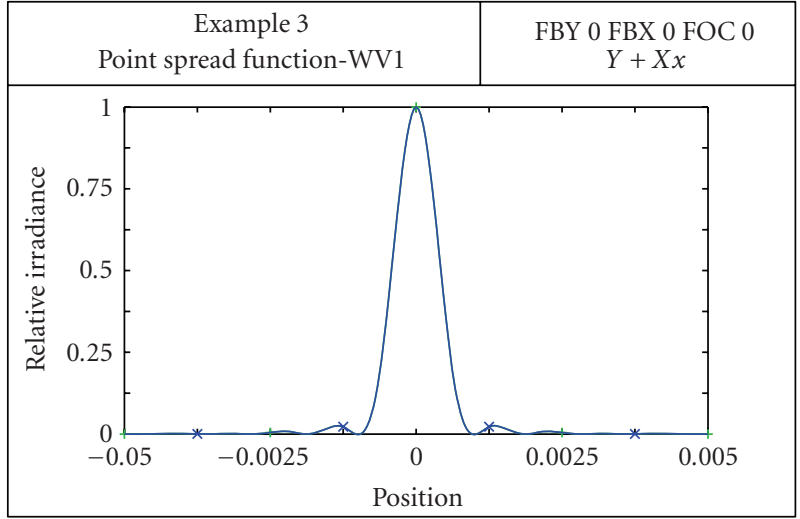

FIgURE 5: The point spread function of the example 3.

TABle 2: Coefficients value for the example 1.

\begin{tabular}{lcc}
\hline Coefficients & Aperture height $(\mathrm{mm})$ & Value \\
\hline$a_{2}$ & Paraxial & $-0.005 \mathrm{~mm}^{-1}$ \\
$a_{4}$ & $(25)(1)=25$ & $1.180409 \times 10^{-6} \mathrm{~mm}^{-3}$ \\
$a_{6}$ & $(25)(0.7746)=19.363$ & $-1.270732 \times 10^{-10} \mathrm{~mm}^{-5}$ \\
\hline
\end{tabular}

TABle 3: Coefficients value for the example 2.

\begin{tabular}{lcc}
\hline Coefficients & Aperture height $(\mathrm{mm})$ & Value \\
\hline$a_{2}$ & Paraxial & $-0.005 \mathrm{~mm}^{-1}$ \\
$a_{4}$ & $(25)(1)=25$ & $1.194430 \times 10^{-6} \mathrm{~mm}^{-3}$ \\
$a_{6}$ & $(25)(0.9137)=22.842$ & $-1.684803 \times 10^{-10} \mathrm{~mm}^{-5}$ \\
$a_{8}$ & $(25)(0.7746)=19.363$ & $3.602457 \times 10^{-14} \mathrm{~mm}^{-7}$ \\
$a_{10}$ & $(25)(0.555)=13.875$ & $-5.856954 \times 10^{-14} \mathrm{~mm}^{-9}$ \\
\hline
\end{tabular}

can be represented only for the first and second terms, and combining these terms, the spherical aberration of the edge can be corrected. Then, the peak of the spherical aberration residual occurs when $y$ is equal to the marginal $y_{m}$ multiplied by $\sqrt{3 / 5}=0.7746$. This analysis is similar to Kingslake [8]. The difference is that the defocus term is not considered here.

It is possible to correct the residual spherical aberration by using the third term of the expansion (9), but now the ray aberration curve has two opposite peaks above and below the 0.7746 zone. The zones with maximum and minimum residuals fall at values of $y$ given by $y / y_{m}=0.5550$ or 0.9137 (see Figure 2).

If we consider f/numbers to be small, we should correct the spherical aberration residual, and its peaks fall at values $y=y_{m}(0.5550)$ or $y_{m}(0.9137)$; then we need fourth and fifth term to correct these other $y$ 's, now the ray aberration curve has two opposite peaks above and two below of 0.5550 and 0.9137 zones. The zones with maximum and minimum residuals fall at values of $y$ given by $y / y_{m}=$ $0.9681,0.8505,0.6661$, or 0.3740 , Figure 7 . This analysis can continue because the expansion (9) is infinity.

The points for Kingslake analysis are $y / y_{m}=1,0.8880$, 0.7071 , and 0.4597 , and for our analysis $y / y_{m}=1,0.9137$, 0.7746 , and 0.5550 . 
TABle 4: Coefficients value for the example 3.

\begin{tabular}{lcc}
\hline Coefficients & Aperture height $(\mathrm{mm})$ & Value \\
\hline$a_{2}$ & Paraxial & $-0.005 \mathrm{~mm}^{-1}$ \\
$a_{4}$ & $(25)(1)=25$ & $9.106091 \times 10^{-7} \mathrm{~mm}^{-3}$ \\
$a_{6}$ & $(25)(0.9137)=22.842$ & $-1.941142 \times 10^{-10} \mathrm{~mm}^{-5}$ \\
$a_{8}$ & $(25)(0.7746)=19.363$ & $5.218183 \times 10^{-14} \mathrm{~mm}^{-7}$ \\
$a_{10}$ & $(25)(0.555)=13.875$ & $-9.916744 \times 10^{-18} \mathrm{~mm}^{-9}$ \\
\hline
\end{tabular}

The number of $y$ that must be corrected for each optical system depends on the optical system tolerances, for example, with one value of $y\left((1)\left(y_{m}\right)\right)$, we correct a lens with $\mathrm{f} /$ number bigger than $\mathrm{f} / 5$; with two different values of $y\left((1)\left(y_{m}\right)\right.$ and $\left.(0.7746)\left(y_{m}\right)\right)$, we correct a lens with $\mathrm{f} /$ number bigger than $\mathrm{f} / 2$; with four different values of $y\left((1)\left(y_{m}\right),(0.7746)\left(y_{m}\right),(0.5550)\left(y_{m}\right)\right.$, and $\left.(0.9137)\left(y_{m}\right)\right)$, we correct a lens with $\mathrm{f} /$ number bigger than $\mathrm{f} / \mathrm{1}$, but only the designer should decide the correction that he needs.

\section{Results}

We have proposed a general expression to compute the phase coefficients. Now, we will show how theses coefficients minimize the spherical aberration with some numerical examples. All examples considered in this section have the diffracted order $m=1$.

4.1. Example 1. In this example, we consider that the diffractive surface is on a spherical surface (the last surface of the system) with $50 \mathrm{~mm}$ of diameter aperture, numerical aperture 0.375 , object distance $200 \mathrm{~mm}$, and $\lambda=0.587 \mu \mathrm{m}$.

In Table 1, other characteristics of the refractivediffractive lens are shown.

We must trace light rays until the last surface, and then we can calculate all constants of (8).
The number of rays traced depends on the number of coefficients. In this example, we use two coefficients, and we get the next equations system

$$
\left[\begin{array}{cc}
6.340530 E+4 & 6.130961 E+7 \\
2.924391 E+4 & 1.6731 E+7
\end{array}\right]\left[\begin{array}{l}
a_{4} \\
a_{6}
\end{array}\right]=\left[\begin{array}{l}
0.067053 \\
0.032394
\end{array}\right] .
$$

We have solved (8) to compute the phase coefficients for two different pupil positions on the surface; they are shown in Table 2.

Figure 2 shows the spherical aberration of the refractivediffractive lens; the graphics were obtained using the commercial optical design program "OSLO" [9].

We can see in the graphic a maximum transversal spherical aberration of about $0.004 \mathrm{~mm}$, having zeros on two pupil positions. This is because we had computed two coefficients for the system. The corresponding Strehl Ratio is of about 0.151 .

In the Figure 2, FBY and FBX are the fractional object coordinates, and WV1 is the wavelength $(\lambda=0.587 \mu \mathrm{m})$ for the evaluation.

4.2. Example 2. We consider the same optical system but now using four phase coefficients. Solving the next equations system

$$
\left[\begin{array}{cccc}
6.34053 E+4 & 6.130961 E+7 & 5.269617 E+10 & 4.246204 E+13 \\
4.821205 E+4 & 3.869253 E+7 & 2.760235 E+10 & 1.84602 E+13 \\
2.924391 E+4 & 1.6731 E+7 & 8.50856 E+9 & 4.056593 E+12 \\
1.071387 E+4 & 3.119059 E+6 & 8.071391 E+8 & 1.958143 E+11
\end{array}\right]\left[\begin{array}{c}
a_{4} \\
a_{6} \\
a_{8} \\
a_{10}
\end{array}\right]=\left[\begin{array}{l}
0.067053 \\
0.051953 \\
0.032394 \\
0.012299
\end{array}\right],
$$

we obtain phase coefficients which are show in Table 3.

In Figure 3, we can see a maximum traversal spherical aberration of the refractive-diffractive lens of about $0.00005 \mathrm{~mm}$, having zeros on four pupil positions. The reason is that we had computed four coefficients for this system. The corresponding Strehl Ratio is of about 1. Figure 3 also shows the difference between the points proposed by Kingslake [6] and our selected points. It can be seen that the points suggested in this paper to correct the spherical aberration are slightly better than the Kingslake points.
4.3. Example 3. Now we consider the same optical system but the diffractive surface on a hyperbolic surface (last surface) with conic constant, diameter aperture $K=$ $-4.654,50 \mathrm{~mm}$, numerical aperture of 0.375 , object distance of $200 \mathrm{~mm}$, and $\lambda=0.587 \mu \mathrm{m}$. We must trace rays to the hyperbolic surface because in this way we can calculate all constants of (8) for this example. We use four phase coefficients to solve the following equations system: 


$$
\left[\begin{array}{cccc}
6.422962 E+4 & 6.209924 E+7 & 5.336845 E+10 & 4.299859 E+13 \\
4.86619 E+4 & 3.905076 E+7 & 2.785591 E+10 & 1.862844 E+13 \\
2.939127 E+4 & 1.681484 E+7 & 8.550953 E+9 & 4.076689 E+12 \\
1.072902 E+4 & 3.123454 E+6 & 8.082727 E+8 & 1.960884 E+11
\end{array}\right]\left[\begin{array}{c}
a_{4} \\
a_{6} \\
a_{8} \\
a_{10}
\end{array}\right]=\left[\begin{array}{c}
0.048792 \\
0.038001 \\
0.023906 \\
9.203867 E-3
\end{array}\right]
$$

In the Table 4 are the new coefficients for this optical system. Figure 4 shows the aberration of this refractive-diffractive lens.

We can see again a very small spherical aberration, and its maximum value is of around $2 \times 10^{-5} \mathrm{~mm}$. It has 4 zeros because we have used 4 phase coefficients. The irradiance distribution corresponding to this system is shown in Figure 5.

Our proposed method is also for flat surfaces. We only use zero for the angle between the normal to the surface and optical axis in (4), and then we obtain the grating (3) for a flat surface. Then, we can use the procedure that we used in the previous examples.

If the designer wants to use the first surface, the conjugates must be changed, and then the method proposed can be applied.

\section{Conclusions}

We have established a new exact method to correct the spherical aberration for any optical system using diffractive lenses; this method makes use of the general grating equation and exact ray trace. With our method, we can decide how many zeros the spherical aberration should have and fix its position in the exit pupil. The method can only be applied to the first and last surface of the optical system.

We also have proposed some heights to correct the spherical aberration and how many rays must be traced depending on the f/number.

In the first and second examples, we have shown that we can have a high control of spherical aberration, minimized at points on the surface where we have wanted. Also, we have shown that our method is valid for any rotationally symmetrical surface.

In general, spherical aberration will have as many zeros as the coefficients we calculate. It is very important to see that in order to minimize spherical aberration, we use only as many coefficients as necessary.

Finally, to calculate the coefficients, we only use the analytic-numerical method. The calculations are exact, simple, and quick. A process of optimization is not required.

\section{References}

[1] N. Davidson, A. A. Friesem, and E. Hasman, "Analytical design of hybrid diffractive-refractive achromats," Applied Optics, vol. 32, no. 25, pp. 4770-4774, 1993.

[2] V. A. Soifer, Methods for Computer Design of Diffractive Optical Elements, John Wiley \& Sons, New York, NY, USA, 2002.

[3] D. A. Buralli and G. M. Morris, "Design of diffractive singlets for monochromatic imaging," Applied Optics, vol. 30, no. 16, pp. 2151-2157, 1991.
[4] J. Castro-Ramos, S. Vázquez-Montiel, J. Hernández-De-LaCruz, O. García-Lievanos, and W. Calleja-Arriaga, "Diffractive optics: a review of the optical systems design and construction using diffractive lenses," Revista Mexicana de Fisica, vol. 52, no. 6, pp. 479-500, 2006.

[5] H. P. Herzig, "Design of refractive and diffractive microoptics," in Micro-Optics: Elements, Systems, and Applications, S. Martellucci and A. Chester, Eds., pp. 23-33, Plenum Press, New York, NY, USA, 1997.

[6] M. Young, "Zone plates and their aberrations," Journal of the Optical Society of America, vol. 62, no. 8, pp. 972-976, 1972.

[7] R. W. Meier, "Magnification and third-order aberrations in holography," Journal of the Optical Society of America, vol. 55, pp. 987-992, 1965.

[8] R. Kingslake, "Spherical aberration," in The Lens Design Fundamentals, chapter 5, pp. 114-115, Academic Press, New York, NY, USA, 1978.

[9] Lambda Research Corporation, OSLO Optics Software for Layout and Optimization, Optics Reference, Version 6.1, Littleton, Mass, USA, 2001. 

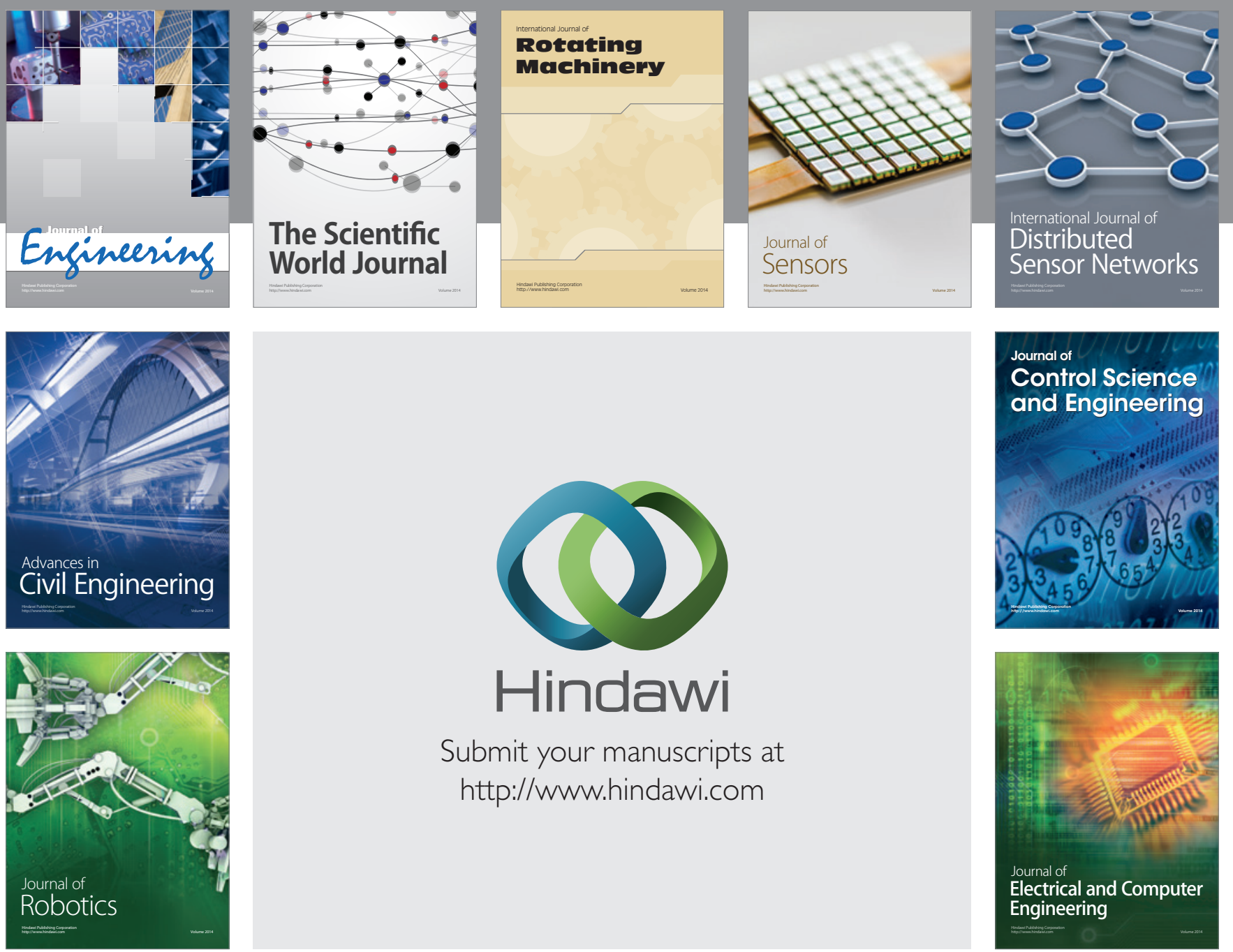

Submit your manuscripts at

http://www.hindawi.com
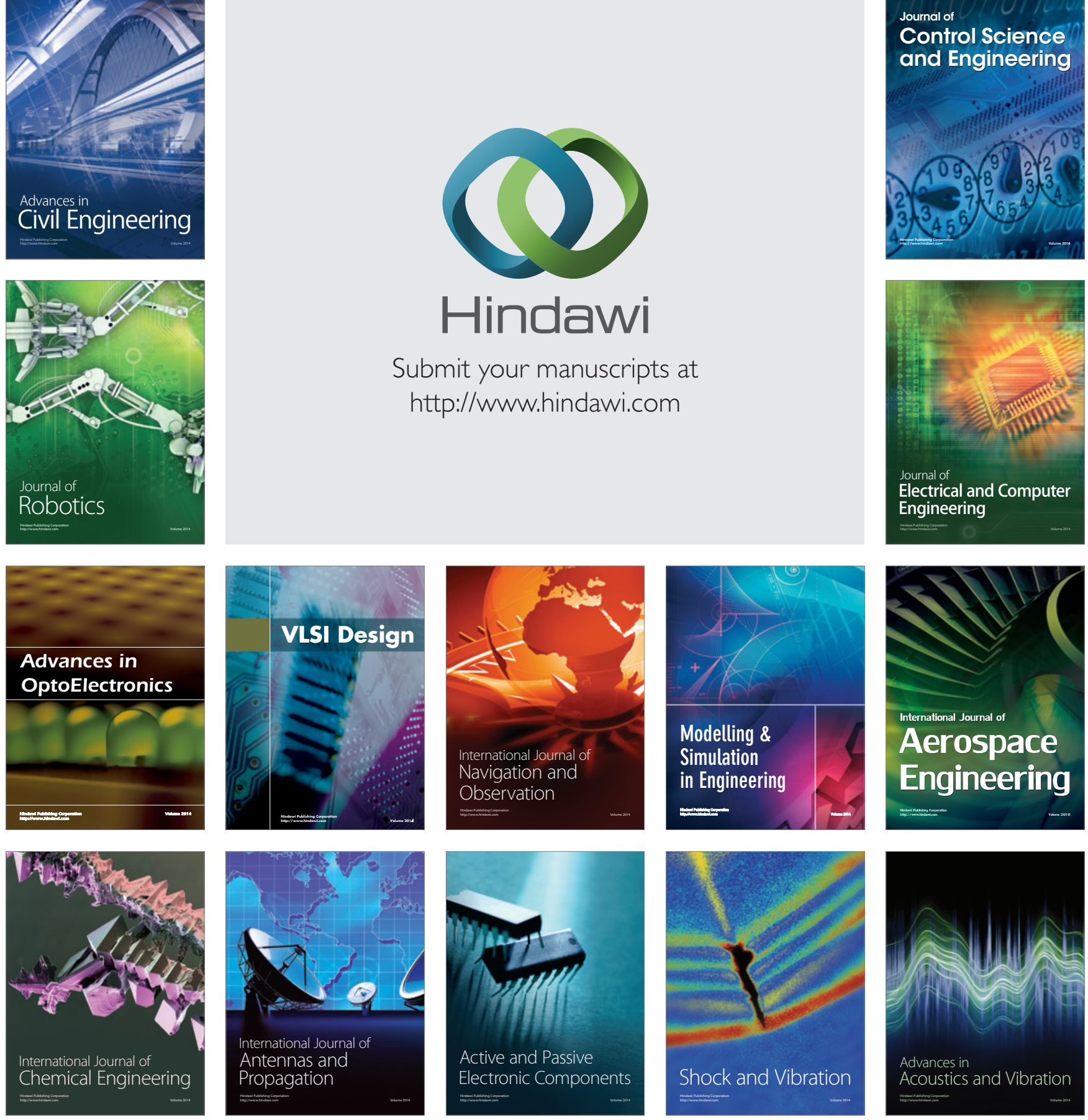